\title{
Sleep and wake disturbances following traumatic brain injury
}

Catherine Duclos ${ }^{\mathrm{a}}$, Marie Dumont ${ }^{\mathrm{a}}$, Catherine Wiseman-Hakes ${ }^{\mathrm{a}}$, Caroline Arbour ${ }^{\mathrm{a}}$, Valérie Mongrain $^{\mathrm{a}}$, Pierre-Olivier Gaudreault ${ }^{\mathrm{a}}$, Samar Khoury ${ }^{\mathrm{a}}$, Gilles Lavigne, ${ }^{\mathrm{a}}$ Alex Desautels ${ }^{\mathrm{a}}$, Nadia Gosselin*a,b

a Center for advanced research in sleep medicine, Hôpital du Sacré-Coeur de Montréal, 5400, boulevard Gouin Ouest, Montréal, Québec, H4J 1C5, Canada

b Université de Montréal, C.P. 6128, succursale Centre-Ville, Montréal, Québec, Canada

Published in: Pathologie-Biologie (2014), 62(5) : 252-261

doi:10.1016/j.patbio.2014.05.014 


\begin{abstract}
Traumatic brain injury (TBI) is a major health concern in industrialised countries. Sleep and wake disturbances are among the most persistent and disabling sequelae after TBI. Yet, despite the widespread complaints of post-TBI sleep and wake disturbances, studies on their etiology, pathophysiology, and treatments remain inconclusive. This narrative review aims to summarise the current state of knowledge regarding the nature of sleep and wake disturbances following TBI, both subjective and objective, spanning all levels of severity and phases postinjury. A second goal is to outline the various causes of post-TBI sleep-wake disturbances. Globally, although sleep-wake complaints are reported in all studies and across all levels of severity, consensus regarding the objective nature of these disturbances is not unanimous and varies widely across studies. In order to optimize recovery in TBI survivors, further studies are required to shed light on the complexity and heterogeneity of post-TBI sleep and wake disturbances, and to fully grasp the best timing and approach for intervention.
\end{abstract}

Keywords: traumatic brain injury, sleep, sleep-wake disturbances, insomnia, hypersomnia, circadian rhythms 


\section{Introduction}

Traumatic brain injury (TBI) is the leading cause of mortality and invalidity among young adults in industrialized countries, with an incidence estimated at over 600 per 100000 individuals [1,2]. This high incidence represents a major public health concern since TBI often results in long-term physical, cognitive and psychological sequelae that interfere with general functioning and return to work or school.

Sleep-wake disturbances, particularly fatigue, insomnia and hypersomnia, are among the most prevalent and persistent sequelea reported after TBI [3,4]. They have been consistently reported among TBI survivors across all levels of severity, from the acute stage to several years post-injury [5-7]. Yet, the pathophysiology of post-traumatic sleep-wake disturbances is still poorly understood.

The purpose of this narrative review is to describe and appraise the current state of knowledge regarding sleep-wake disturbances following TBI across all levels of severities, spanning the continuum of recovery from the acute stage (first weeks post-injury) to the chronic stage, including years post-injury. A second goal is also to describe the possible causes of sleep-wake disturbances following TBI, as well as pharmacologic and nonpharmacologic treatment options for the management of sleep-wake disturbances post-injury. Finally, the importance of sleep for cognitive and functional recovery will be discussed, and general directions for future study perspectives will be provided.

For the purpose of this literature review, the acute phase of TBI refers to the first 6 months post-injury, which constitutes the period immediately following TBI and the early phase of recovery. The chronic phase of TBI will refer to the period following the acute phase, spanning from 6 months to several years post-injury, regardless of the presence of symptoms.

\section{Diagnosis and general consequences of TBI}

Traumatic brain injury occurs when an external force causes an alteration in brain functions such as decreased level of consciousness, loss of memory, neurological deficits or any alteration in mental state at the time of the injury [8]. The diagnosis of TBI necessarily 
involves a severity assessment [9]. Globally, mild TBI (mTBI) is characterized by a short loss of consciousness $(<30 \mathrm{~min})$, and/or a short post-traumatic amnesia $(<24 \mathrm{~h})$, a Glasgow Coma Scale (GCS) score [10] between 13 and 15 [1]. Sports-related brain injuries with an alteration in mental state are generally referred to as "concussion". Moderate and severe TBI are typically associated with longer loss of consciousness, a GCS score equal or lower than 12, and PTA longer than $24 \mathrm{~h}$. No hospital admission is generally required for mTBI, while moderate and severe TBI often necessitate hospitalisation in the intensive care unit due to the presence of cerebral haemorrhages, contusions and intracranial hypertension [11].

The most frequent causes of TBI are motor-vehicle accidents, falls, assault, recreational and sport-related injuries, and work accidents [12,13]. The principal factors known to increase the risk of sustaining a TBI are age (15-24 years old) and gender (male); other factors are alcohol or drug addiction, low socioeconomic status, and low education [14].

The extent of functional and cognitive deficits observed in the acute period is highly variable among TBI patients and depends on several factors, such as location of focal lesions $[15,16]$, severity of diffuse axonal injury, length of PTA [15,17], age[16, 18], education [16], and preexisting conditions [19]. Despite the variability of deficits, they can be categorized in relation to their influence on cognition, social behaviors, psychological status, and somatic symptoms.

In fact, arousal and alertness impairments, reduced information processing speed, impaired memory, executive dysfunctions, impaired communication, and reduced selfawareness are among the most frequent cognitive deficits observed [20]. Neurobehavioral impairments such as impulsivity, irritability, disinhibition, mutism, confusion and confabulatory communication, and apathy can be observed, particularly in those with more severe injuries and or in the acute stage of injury [21]. Psychological sequelae, such as irritability, anxiety, and depressed mood can be observed in $\geq 50 \%$ of individuals in the first 6 months following severe TBI [22]. These affective symptoms remain prevalent among 16$48 \%$ of patients over the two years post-injury, and are reported at many years post injury (>10yrs) in those with chronic sleep-wake disorders [23]. Finally, somatic symptoms, such as 
headache, dizziness, but also perturbation in sleep are often reported in the hours following TBI [22] and these symptoms remain present in $23-65 \%$ of patients two years after TBI onset.

Recovery from symptoms usually takes place within 1 to 3 months in mTBI, while $85 \%$ of the improvement in functioning occurs in the first six months and improvement continues until one year after moderate to severe TBI [24]. Unfortunately, impairments in these symptoms, including in those related to sleep disturbances, persist over one year in 50\% of moderate-severe TBI patients [25], affecting their autonomy, productivity, and their quality of life [26].

\section{Sleep disturbances following TBI}

\subsection{Changes in sleep quality and quantity}

Complaints of sleep loss and poor sleep quality are common following TBI. These comprise complaints of difficulties initiating and maintaining sleep, frequent arousals, and early awakenings. In the following section, prevalence of poor sleep quality and related polysomnographic findings will be described for mTBI and for the moderate-severe TBI population separately. For each TBI severity population, results for the acute and chronic phases will be reported.

\subsubsection{Acute stage of $\mathrm{mTBI}$}

According to Chaput et al. [27], sleep complaints are present in the first days and weeks following mTBI. In fact, $13.3 \%$ of the 443 patients included in their study reported sleep complaints on the Rivermead post-concussion symptom assessment questionnaire 10 days post-injury, while this proportion increased to $33.5 \%$ at 6 weeks post-injury. Interestingly, patients with sleep complaints at 10 days post-injury were 2.9 times more likely to experience sleep difficulties at 6 weeks post-injury and were more likely to suffer from irritability, depressive symptoms, and headaches at both 10 days and 6 weeks post-injury, suggesting that acute sleep complaints predict psychological and somatic symptoms among individuals with mTBI. Poor sleep quality was also reported after sports-related concussions where athletes who had suffered at least one concussion (4.4 \pm 3.8 months since injury) 
complained of worst sleep quality, more severe sleep disturbances and poorer daytime functioning on the Pittsburgh Sleep Quality Index when compared with healthy control athletes [28].

Although a high proportion of patients have sleep complaints in the acute phase of their mTBI, heterogeneous results were obtained on objective measures of sleep such as polysomnography (PSG). In fact, several authors found no differences in sleep macroarchitecture between mTBI patients and controls in the acute stage of the injury [28-30].

Conversely, studies looking at sleep microarchitecture have found promising results. In fact, Rao et al. [29] showed that mTBI patients within 1 week of injury had abnormalities in sleep EEG power spectral analyses when compared to matched controls. More specifically, mTBI patients had lower delta power, but higher alpha and beta power in non-rapid eye movement (NREM) sleep. A more recent study conducted by Khoury et al. [31] compared 24 mTBI patients ( $45 \pm 22.7$ days post-injury) with post-traumatic sleep complaints to 18 controls on quantitative EEG during sleep. Overall, results showed that patients reported a worse sleep quality for their in-laboratory sleep recording compared with control subjects. Moreover, mTBI patients had significantly longer sleep latency $(17.8 \pm 15.3 \mathrm{~min}$ vs. $8.9 \pm 5.7 \mathrm{~min})$, which was within normal range $(<20 \mathrm{~min})$ despite their complaints of poor sleep, and lower sleep efficiency $(89.2 \pm 7.5 \%$ vs. $93.7 \pm 5.7 \%)$. Lower delta in REM sleep and higher beta and gamma power in NREM sleep were also observed among the mTBI group compared to the control group, and pain was the main factor associated with abnormal EEG power during sleep. This microarchitecture sleep pattern characterised by reduced power of low frequency bands and increased power of high frequency bands may represent hyperarousal among mTBI individuals and was previously documented among insomniac patients [32].

\subsubsection{Chronic stage of $\mathrm{mTBI}$}

Subjective sleep complaints have also been widely documented in long-term mTBI, the most pervasive of which are insomnia, sleep fragmentation, and early morning awakenings. $[3,33,34]$ Beetar et al. [33] conducted a retrospective chart review in order to compare the incidence of sleep complaints in symptomatic mTBI. The study included 202 TBI patients 
(127 mild and 75 moderate-severe) $23.9 \pm 21.2$ months post-injury, who were compared to a group of non-TBI neurologic patients. Insomnia complaints were reported by $65.3 \%$ of all mTBI patients. Overall, insomnia complaints were significantly more prevalent in the TBI group compared with the non-TBI neurologic patients (56.4\% vs. $30.9 \%$ ), and more frequent among mTBI than moderate-severe TBI $(65.4 \%$ vs. $41.3 \%)$. Sleep complaints were still present in $23.7 \%$ of mTBI patients at least five years post-injury. In another study aiming to delineate which factors predict suboptimal outcome after TBI, Clinchot et al. [34] found that approximately $75 \%$ of the $145 \mathrm{mTBI}$ patients included in their study reported sleep disturbances one year post-injury, the most common of which was waking up too early. Globally, these studies indicate that complaints of poor sleep are found in approximately 65$75 \%$ of chronic mTBI patients.

With the conflicts in Afghanistan and Iraq, the military population has recently sparked the interest of TBI researchers, even though the mechanism of injury often differs from that of TBI in civilians. One study [35] evaluated 116 soldiers with combat-related blunt and blast TBI ( $84.5 \%$ mild) $16.1 \pm 11.5$ months post-injury using directed questioning regarding sleep complaints. Nearly all (97.4\%) participants reported sleep complaints. More specifically, poor sleep quality was reported by $81.9 \%$ of participants, and $54.3 \%$ reported sleep fragmentation. According to the study by Bryan [36], the incidence of insomnia increased with TBI frequency in the military population, from $5.6 \%$ for no TBIs, $20.4 \%$ for single TBI, and $50.0 \%$ for multiple TBIs.

In the chronic phase of mTBI, studies that have objectively measured sleep through PSG have generally been able to corroborate the pervasive self-reported complaints of disturbed sleep identified in the abovementioned studies. More specifically, studies using PSG have shown that those with mTBI and persistent symptoms have less efficient sleep, shorter REM onset latency, longer sleep onset latency, shorter total sleep time, a decrease in REM sleep and an increase in stage N2 sleep, when compared to healthy age- and sex-matched controls [37,38]. In the abovementioned study performed among soldiers [35], PSG findings revealed sleep fragmentation, with an average sleep efficiency of $86.3 \% \pm 12.1 \%$ and a mean 
total arousal index of $17.7 \pm 11.5$ events per hour. Insomnia was present in $55.2 \%$ of their subjects based on DSM-IV diagnostic criteria.

Importantly however, to our knowledge, there have not been any studies to date that have objectively investigated the sleep of all those with mTBI; current studies are limited to recruitment of participants with self-reported sleep complaints. Such a study would be invaluable in order to elucidate on the role of mTBI in generating structural sleep changes, and on the factors associated with the presence and absence of sleep complaints.

\subsubsection{Acute stage of moderate and severe TBI}

Clinical observations in acute care settings suggest that sleep disturbances appear in the first weeks after TBI, where patients present insomnia, an inability to stay awake for a few consecutive hours during the day, and/or altered sleep-wake cycles. To date, a few studies have investigated acute sleep-wake disturbances during the period of hospitalisation, including in the intensive care unit (ICU), regular units of trauma center and in rehabilitation centers during the early period of rehabilitation. A recent study conducted by our group aimed to measure the rest-activity cycle when patients were hospitalised in the intensive care unit and regular wards, looking specifically at the consolidation of rest and activity periods, or patients' ability to sustain activity during the day and rest during the night [39]. Using 10-day actigraphy recordings in $16 \mathrm{TBI}$ patients, the study showed severe fragmentation of the restactivity cycle, reflecting fragmentation of sleep and wake episodes. Using a ratio of daytime activity to $24 \mathrm{~h}$ activity $\geq 80 \%$ to denote rest-activity cycle consolidation, we were able to show that the rest-activity cycle was consolidated only $46.6 \%$ of all days, but that a significant linear trend of improvement was found over time. Worse sleep-wake cycle consolidation and evolution were associated with higher TBI severity, and longer duration of ICU and hospital stay. Patients with more rapid return to a consolidated rest-activity cycle were more likely to emerge from PTA and to have lower disability at hospital discharge. Another preliminary study conducted by our group compared the sleep architecture of 6 moderate-severe TBI (4 males; $25 \pm 11.3 \mathrm{yrs}$ ) during their hospital stay to that of 11 healthy controls ( 7 males; $25 \pm 10.5$ yrs) [40]. The TBI patients underwent 24-h bedside ambulatory PSG, while controls underwent in-laboratory PSG. Our results indicated that TBI patients had more fragmented 
sleep characterised by greater total wake duration, $(159 \pm 104.7 \mathrm{~min}$ on average as compared to $53.7 \pm 47.0$ for controls) and a higher number of arousals ( $49.7 \pm 35.4$ on average as compared to $20.2 \pm 7.8$ for control subjects). Sleep efficiency for those with TBI was $74 \pm 15.9 \%$ (on average) in comparison to $88 \pm 9.3 \%$ for controls.

Studies carried out during early rehabilitation also point to a high prevalence of sleepwake disturbances. One research group conducted a prospective observational study of 31 patients with moderate-severe TBI admitted to an inpatient rehabilitation unit, and found that $68 \%$ had disturbed nighttime sleep, defined as two or more hours awake during the night, as measured by hourly nurse observations [41]. This same group conducted another study in which they used actigraphy on 14 moderate-severe TBI patients for the duration of their stay in a rehabilitation unit, between 9 to 23 days post-injury [42]. Overall, $78 \%$ of patients had a mean 1-week sleep efficiency that was severely impaired $(\leq 63 \%)$. Patients who had cleared PTA prior to rehabilitation admission had significantly better sleep efficiency than patients with ongoing PTA. Nakase-Richardson et al. [43] conducted a prospective observational study on primarily severe TBI patients, using item one of the Delirium Rating Scale-Revised-98 (DelRS-R98) to classify the severity of sleep-wake cycle disturbance as none, mild, moderate, or severe. Results showed that mild to severe sleep disturbances were present among $84 \%$ of patients upon admission to a rehabilitation hospital, and persisted for $66 \%$ of patients one month post-injury. The presence of sleep disturbance at one month post-injury had a significant predictive value on the duration of PTA.

\subsubsection{Chronic Moderate-Severe TBI}

Studies investigating subjective sleep complaints in the chronic phase of moderatesevere TBI patients point to frequent complaints of TBI-induced changes in sleep quality [3,34,44-48], changes in bedtime[47,48], longer sleep onset latency [46-48], more nocturnal awakenings [47], and symptoms of insomnia [3,49]. Overall, subjectively-reported sleep changes have been reported in 42 to $80 \%$ of TBI patients, though perception of one's condition may be altered by persistent cognitive deficits among this population, leading to and underreporting of complaints [34,45-48]. In a meta-analysis of 1706 TBI survivors documented across 21 studies utilizing both objective and self-report measures, Mathias and 
Alvaro [50] reported that TBI survivors were likely to suffer from insomnia (observed in 50\% of TBI patients), difficulty maintaining sleep (50\%), poor sleep efficiency (49\%), early morning awakenings (38\%), difficulty with sleep initiation (36\%) and nightmares (27\%).

Studies that have used PSG to objectively measure sleep in chronic moderate-severe TBI patients have shown impaired sleep efficiency [44,51], an increase in slow wave sleep [52], and excessive nocturnal awakenings [51,53]. Interestingly, Mathias and Alvaro [2012] reported that objective sleep measures identified more sleep-wake problems than self-report measures on problems with sleep maintenance, sleep efficiency, and awakening [50].

Ouellet et al. [3] used a detailed questionnaire to assess the sleep quality and fatigue of 452 community-based TBI patients (all severity; 83.2\% moderate-severe). Over $50 \%$ of patients reported symptoms of insomnia, and $29.4 \%$ fulfilled the diagnostic criteria for an insomnia syndrome. In a cross-sectional study including 121 TBI survivors 2 years postinjury, Cantor et al. [49] found a similar prevalence of insomnia syndrome (24\%) using the DSM-IV and ICSD diagnostic criteria. Since these patients were not recruited from a sleep clinic or according to the presence of sleep complaints, these results suggest that a high proportion of TBI patients have sleep problems but do not consult a sleep clinic, leaving these problems untreated.

Although studies that have utilized objective sleep measures have also identified various sleep disturbances in moderate-severe TBI patients, one study reported no significant differences on PSG variables between 22 moderate and severe TBI survivors tested $53.0 \pm$ 37.1 months post-injury compared to 22 controls [54]. However, patients in this particular study were community-based participants who were not consulting a sleep clinic, as opposed to most other PSG studies on moderate-severe TBI patients. Because time since injury ranged from 1 to 11 years, the authors suggested that objectively-measured sleep alterations could become gradually attenuated over time, which could explain why their study showed no significant differences between TBI patients and controls. Another study sought to compare subjective sleep complaints to objective measures in 14 TBI patients 21 months post-injury on average with an insomnia complaint and 14 healthy controls [53]. Even though significant differences were found on all subjective sleep measures, no PSG variables were significantly 
different between groups. These findings highlight an important issue for consideration when comparing research across the literature, as some studies recruit on the basis of self-report of sleep problems, or those seeking help for sleep problems, while others, such as the study by Beaulieu-Bonneau [54], do not.

\subsection{Hypersomnia and excessive daytime sleepiness}

Hypersomnia, or increased sleep need per 24-h, is a widespread condition following TBI that is often associated with excessive daytime sleepiness (EDS), though EDS may be a result of insomnia or poor nocturnal sleep. One study group has suggested that pleiosomnia, or an increased need for sleep of at least 2 hours per 24-h, was a more accurate term to reflect an increased pressure to sleep following TBI [52]. In the following section, hypersomnia and EDS are presented for mild and moderate-severe TBI separately.

\subsubsection{Mild TBI}

Watson et al. [55] used the Sickness Impact Profile, a detailed health status questionnaire that measures behavioral changes due to illness, to assess sleepiness at one month post-injury, for which they evaluated 348 TBI patients (78\% mild TBI), 132 noncranial trauma controls, and 102 trauma-fee controls. The study showed that at one month post-injury, a significantly greater proportion of TBI subjects $(55 \%)$ endorsed one or more sleepiness items of this questionnaire, as opposed to non-cranial trauma controls (41\%) and trauma-free controls (3\%). A greater number of TBI subjects endorsed each of the four sleepiness items than did both control groups. Among the military population, complaints of EDS were identified as being widely pervasive, and reported by $85.2 \%$ of participants [35].

Despite this high prevalence of daytime sleepiness, very few studies investigated hypersomnia and EDS using objective measures among the mTBI population. One study used the Multiple Sleep Latency Test (MSLT) to compare the sleepiness mTBI patients (12 months to 21 years post-injury) who had been referred to a sleep lab to that of healthy matched controls. The MSLT tests for excessive daytime sleepiness by measuring sleep latency during five scheduled daytime naps, each separated by two hours of wake. The authors were able to 
confirm patients' complaints and showed that mTBI patients had significantly greater number of sleep entrance episodes, and a significantly shorter time to fall asleep [38].

Gosselin et al. [28] examined the spectral analysis of the waking EEG in 11 concussed athletes as compared to healthy control athletes. Their results showed increased delta and reduced alpha EEG activity during wakefulness, while no modifications were found during sleep despite the high frequency of poor sleep quality complaints, suggesting that sport-related concussions are associated with wakefulness dysfunctions rather than sleep disturbances per se. The authors proposed that this waking EEG pattern may represent impaired vigilance among concussed athletes.

\subsubsection{Moderate-severe TBI}

Studies carried out one to three months post-injury have shown that daytime sleepiness is highly present following moderate-severe TBI [55,56]. While Watson et al. [55] found that greater sleepiness was associated with greater TBI severity, Rao et al. found no such association [56]. Studies investigating subjective daytime complaints in the chronic phase of moderate-severe TBI patients point to more frequent and longer daytime napping [48,54,57], and EDS [30,48,51,57,58].

Among studies that have recruited patients with sleep complaints, the study conducted by Verma et al. [51] showed that 30 of the 60 TBI patients $(60 \%$ moderate-severe according to the Global Assessment of Functioning scale) reported hypersomnia as their presenting complaint. Overall, 28 patients had an elevated Epworth Sleepiness Scale (ESS) score higher than 11, pointing to EDS. Of these 28 participants who underwent MSLT, 15 had a mean sleep onset latency $<5$ min, confirming their complaints. Sommerauer et al. [52] conducted a casecontrol study compared 36 consecutively admitted TBI patients (13 mild; 23 moderate-severe) who reported pleiosomnia, defined as an increase need for sleep of at least $2 \mathrm{~h}$ per $24 \mathrm{~h}$, to healthy controls, using detailed history, sleep logs, actigraphy, nocturnal PSG, and the MSLT. They found that EDS was highly prevalent (42\%), as was the number of patients taking daytime naps $(47 \%)$. The authors also found that nocturnal total sleep time was increased in 
TBI patients, though patients underestimated their need for sleep. This finding led the authors to conclude that pleiosomnia may be even more frequent than previously reported.

Other studies that have recruited their TBI patients from the community, without considering the presence of sleep complaints, and yet have also found daytime sleepiness [4,30]. Baumann et al. [30], who evaluated 65 patients 6 months post-injury, and Castriotta et al. [4], who evaluated 87 TBI patients ( $8 \%$ mild; $59 \%$ moderate-severe; remaining TBI of unknown severity) $64.3 \pm 117.7$ months post-injury, both used PSG, and MSLT to assess objective sleepiness in TBI patients. In their studies Baumann et al. [30] and Castriotta et al. [4], found that hypersomnia was present in 25 and 11\%, respectively. Using a criteria of sleep latency $<5$ min on the MSLT to represent EDS, Baumann et al. [30] also showed that $25 \%$ of the study sample had objective EDS, compared to $1-6 \%$ in the general population [59]. This prevalence of objective daytime sleepiness was close to that of subjective daytime sleepiness, as measured by the ESS, which was found in $28 \%$ of the sample. Twelve patients (18\%) reported regular daytime napping ( $\geq 3$ per week). Though Baumann et al. [30] found that hypersomnia was associated with severe TBI, Castriotta et al. [4] found no association between daytime sleepiness and injury severity or time since injury.

Despite several studies having highlighted the increase in EDS following TBI, the previously mentioned study by Beaulieu-Bonneau [54] revealed no significant differences on objective sleepiness, as measured by MSLT, between 22 moderate and severe TBI survivors tested $53.0 \pm 37.1$ months post-injury and 22 matched controls.

\section{Other sleep-wake disorders common following TBI}

Overall, studies that have objectively measured sleep using PSG have also found other sleep disorders in chronic moderate-severe TBI. Among them, the study by Verma et al. [51], included 60 TBI patients (40\% mild, $60 \%$ moderate-severe; 3 months to 2 years post-injury) consulting in a sleep clinic and the following sleep disorders have been observed: hypoxia (observed in $70 \%$ of patients), periodic leg movement during sleep (35\%), REM sleep behaviour disorder or increased electromyogram tone during REM sleep (13\%), and sleeponset REM periods, often associated to narcolepsy (5.5\%). Parasomnia were diagnosed in 16 
patients (25\%) and comprised acting out dreams (8\%), sleepwalking (8\%), nightmares (7\%), sleep paralysis (5\%), nocturnal enuresis (5\%), cataplexy (3\%) and nocturnal eating (3\%) [51].

Other studies have recruited their TBI patients in community, without considering the presence of sleep complaints, have also shown that several sleep disorders were present following TBI. When using PSG among those TBI patients (all severity) $64.3 \pm 117.7$ months post-injury, Castriotta et al. [4] found abnormal sleep in $46 \%$ of the study sample, which included obstructive sleep apnea (OSA) (23\%), hypersomnia (11\%), periodic leg movements in sleep (7\%), and narcolepsy (6\%). Among soldiers with TBI, 34.5\% had OSA syndrome [35].

\section{Pathophysiology of sleep-wake disturbances following TBI}

The pathophysiology of post-traumatic sleep-wake disturbances still remains unclear but can possibly be explained by a complex interaction between several physiological, environment, and psychological factors.

\subsection{Brain lesions and brain dysfunctions}

Abnormal neuroimaging results, mostly from computed tomography scanning, are found in approximately 5 to $10 \%$ of mTBI patients [22] and in up to $90 \%$ of patients with severe TBI [60]. Traumatic brain injury also results in more subtle brain damage characterised by decreased synaptic density, and axonal and dendritic degeneration [61]. The cortical and subcortical structures and networks involved in sleep, wake, and circadian rhythmicity may be impaired or damaged in a proportion of TBI patients and possibly explain part of the sleepwake disturbances observed in this population, but no study specifically assessed this association yet.

Baumann [30] found that in $43 \%$ of his study sample, potential causes of sleep and wake disturbances could not be explained by any other factor than the TBI itself (all severity). The possible factors that were considered were sleep-related breathing or movement disorders, narcolepsy or behaviourally induced insufficiency sleep syndrome, substance abuse, demographic characteristics, residual clinical symptoms, or other TBI characteristics. 
Furthermore, it has been shown that patients in the acute phase of moderate to severe TBI have abnormally low levels of hypocretin-1 levels in their cerebrospinal fluid $[30,62]$. Hypocretin-1 (orexin A) is an excitatory neuropeptide produced by the hypothalamus, which is involved in the regulation of arousal. Involvement of the hypocretin system in the acute sleep-wake disturbances of TBI patients is possible, and could be linked to hypersomnia. However, based on currently available research, the hypocretin system may not necessarily explain the high prevalence of chronic sleep-wake disturbances, including insomnia as return to normal values of hypocretin has been observed 6 months post-TBI. Further research is needed to elucidate the underlying pathophysiological mechanisms of chronic sleep-wake disturbances of varying diagnoses (i.e. insomnia, hypersomnia, fragmented sleep, increased sleep need, pleiosomnia).

\subsection{Circadian rhythm disturbances}

One factor that may contribute to sleep-wake disturbances is altered circadian rhythms. The circadian clock, located in the suprachiasmatic nucleus of the hypothalamus, is primarily synchronised to the 24-h environmental day by the light-dark cycle $[63,64]$. Circadian disruption occurs when the main biological clock, located in the hypothalamus, is not synchronised to the 24-h day and/or when it produces a circadian signal too weak to entrain properly the peripheral clocks located in other regions of the brain and body. One of the most obvious manifestations of circadian disruption is a decreased consolidation and abnormal timing of the sleep-wake cycle [65].

Sleep-wake disturbances reported by mTBI patients in the chronic phase of injury may be caused by circadian rhythm dysfunctions that can be manifested as advanced or delayed phase syndrome. Concordant with this hypothesis, Ayalon et al. [66] used actigraphy, salivary melatonin, oral temperature measurement and PSG, to evaluate 42 patients with mTBI and insomnia. The authors founds that $15(36 \%)$ had circadian rhythm sleep disorders ( 8 had a delayed sleep phase syndrome and 7 displayed an irregular sleep-wake pattern).

Alterations in daily rhythms have also been found in chronic moderate and severe TBI, where patients showed decreased evening melatonin production [44,67]. Decreased melatonin 
secretion, however, was not found in another study performed in 10 TBI patients $516 \pm 124.04$ days post-injury and 10 age- and sex-matched controls [68].

Moreover, the immune response triggered by the TBI itself, and possibly other injuries that may accompany it, could contribute to circadian dysregulation following injury. Indeed, increasing evidence points to a bidirectional communication between circadian physiology and immune function [69].

Clock genes may also be dysregulated following TBI. An animal study conducted by Boone et al. [70] found that male rats exposed to fluid-percussion TBI had altered circadian gene expression in both the suprachiasmatic nucleus $(\mathrm{SCN})$ and hippocampus, when compared to sham surgery rats. Bmall and Cryl, both key clock genes, were dysregulated, as was the daily rhythm of locomotor activity, which persistently showed reduced activity in the TBI group, coinciding with the dysregulation of clock genes in the SCN and hippocampus. The authors suggested that a disturbance in the transcriptional-translation feedback loops that modulate circadian timing could be induced by TBI.

During the hospitalisation period following TBI, the hospital environment itself may favour the loss of light/dark circadian cues due to constant lighting [71], which can influence the circadian clock. The fact that patients are bedridden in a constant horizontal posture may also promote daytime sleep, which in turn, also influences nocturnal sleep. A few studies have investigated the circadian rhythms of acute TBI patients hospitalised in the intensive care unit, all of which have pointed to major circadian anomalies, which suggest that brain injury may aggravate circadian rhythms disturbances found among critically ill patients [72-74]. A prospective clinical study conducted by Paul and Lemmer [72] showed an absence of 24-h variation in well-documented markers of circadian rhythms, namely body temperature, plasmatic melatonin and cortisol, heart rate, blood pressure, and spontaneous motor activity in 24 critically ill analgo-sedated patients. The observed circadian rhythm disturbances were more pronounced in the 11 patients with severe brain injury (including 3 moderate-severe TBI patients, 7 patients with subarachnoid hemorrhage following the rupture of an intracerebral aneurysm, and 1 patient with hypoxic brain injury) than in patients without a brain injury. To 
our knowledge, no study has specifically sought out to investigate circadian alterations in the acute phase of mTBI.

\subsection{Endocrine dysfunction}

Endocrine dysfunctions are also common following TBI, especially for endocrine functions emerging from the pituitary, and reported as varying from 15\% to as high as $90 \%$ among moderate-severe TBI patients 2 weeks to several years post-injury [75,76]. These disturbances include hypopituitarism, impaired growth hormone release, hypo/hyperthyroidism, hypothalamic gonadism, and abnormal adrenocortical function. Endrocrine disturbances may be associated with sleep-wake disturbances and fatigue following TBI, as some endocrine changes, such as growth hormone (GH) deficiency and altered cortisol levels, are known to have specific effects on sleep architecture. To test for endocrine dysfunctions, routine blood tests can be carried out to assess hormone levels of the pituitary gland, particularly those of the anterior pituitary (e.g. somatotrophins, corticotropins, thyrotrophins, lactotrophins and gonadotropins), most commonly found to be altered following TBI (see Table 1).

\subsection{Impact of the hospital environment}

In the acute phase post-injury, hospitalised TBI patients are confronted by environmental conditions that are unfavourable to adequate sleep and circadian rhythmicity. Many factors may account for the poor sleep of hospitalised patients, particularly in the ICU environment, where several sources of noise are present, including alarms and conversations from healthcare personnel [77]. In fact, Friese et al. [78] indicated that $36 \%$ of hospitalised TBI patients with sleep complaints pointed out the hospital environment as an important causal factor. Receiving 24-hour care has also been shown to make sleep more difficult [79]. Being under the effects of sedatives, analgesics, narcotics, anticonvulsants and antipsychotics may also influence sleep characteristics [80]. As previously mentioned, the loss of light-dark circadian cues due to constant lighting may also be an environmental factor highly responsible for sleep-wake and circadian disturbances. 


\subsection{Anxiety and depression}

Anxiety and depression have been shown to be highly prevalent after TBI and to have a negative impact on sleep $[3,23,46,47,67]$. Furthermore, those with TBI can suffer from acute post-traumatic stress disorder, a mental disorder that can manifest itself following a psychologically traumatizing event, and can lead to an alteration of sleep and a decrease of slow-wave sleep [81]. Huang et al. [45] recently examined risk factors associated with persistent sleep complaints following TBI (all severity). The authors subdivided the 25 patients with persistent sleep complaints into two groups, comparing those with sleep complaints at 6 and 12 months to those with complaints at 12 months only. The group with complaints at 6 and 12 months had higher levels of depression and post-traumatic distress at both 6- and 12-months post-injury. Conversely, Rao et al. [56] reported that based on structured clinical interview, the presence of insomnia in the acute period following TBI was related to heightened anxiety. With regards to TBI, depression has been associated with reports of sleep changes and worse sleep quality, as well as an increase in nighttime awakenings (subjective and objective) [47,31]. Though anxiety and depression can alter sleep and wake, these studies do not suggest that sleep-wake disturbances are only driven by these effects, but that they may rather occur in parallel.

\subsection{Pain}

Pain is another factor to which sleep disturbances have been partly attributed in several studies $[3,31,33,46,48,67,82]$. In fact, Beetar et al. [33] showed that when patients with pain complaints were removed from his study sample, the prevalence of insomnia complaints was reduced by nearly half, plummeting from $69 \%$ to $38.6 \%$. A recent study linked pain with an increase in rapid EEG frequency bands mostly during REM and slow wave sleep in mTBI (45 \pm 22.7 days post-injury) with pain [31]. TBI patients without pain did not exhibit rapid EEG frequency bands, suggesting that pain may explain, at least in part, poor sleep in this population. 


\section{Management of sleep-wake disturbances}

\subsection{Modifying the hospital and early rehabilitation environments to improve sleep}

Since the environment may increase sleep disturbances in the hospital setting, some environmental factors may be easily modified to promote sleep. For example, providing patients with ear plugs and eye masks, or minimizing staff interventions at night and during naps could enable patients to have a more restorative sleep [77].

Among individuals with circadian disorders (i.e. phase advance or delay), light therapy has been shown to adjust the circadian clock to the environment [83], and has been recognized by the American Academy of Sleep Medicine [84]. Light therapy has previously been used among in the ICU among non-TBI patients, and has been shown not only to improve patients' circadian rhythms, and lead to a swifter recovery [85], but the efficiency of this intervention needs to be evaluated among acute TBI patients.

\subsection{Interventions to improve insomnia}

Prior to treating insomnia, the clinician should screen for other sleep disorders (e.g. restless leg syndrome) as well as medical (e.g. acute and chronic pain) or psychiatric conditions (e.g. anxiety, depression, and posttraumatic stress disorder) that may be associated with, or underlie insomnia (see Table 1). 
Table 1. Screening and interventions for post-TBI insomnia and hypersomnia (list not exhaustive).

\begin{tabular}{|c|c|c|}
\hline & Insomnia & Hypersomnia \\
\hline SCREENING & \multicolumn{2}{|c|}{$\begin{array}{l}\text { Insomnia and hypersomnia may be caused or exacerbated by the following conditions, } \\
\text { which should be ruled out. }\end{array}$} \\
\hline Medical history & $\begin{array}{l}\text { A particular attention should be paid to: } \\
-\quad \text { Acute/chronic pain } \\
\text { - } \quad \text { Current (or withdrawal from) } \\
\text { medication and dosage }\end{array}$ & $\begin{array}{cl}\text { A particular attention should be paid to: } & \text { Neurodegenerative diseases } \\
- & \text { Current medication and } \\
& \text { dosage } \\
- & \text { Weight gain } \\
- & \text { Brain lesions }\end{array}$ \\
\hline $\begin{array}{l}\text { Neuropsychiatric } \\
\text { evaluation }\end{array}$ & $\begin{array}{ll}- & \text { Anxiety } \\
- & \text { Acute/chronic stress } \\
- & \text { Depression (and other mood } \\
& \text { disorders) } \\
- & \text { Posttraumatic stress disorder } \\
\text { - } & \text { Substance abuse disorders }\end{array}$ & $\begin{array}{ll}- & \text { Depression (and other mood } \\
\text { disorders) } & \\
\text { - } & \text { Substance abuse disorders }\end{array}$ \\
\hline Sleep disorders & $\begin{array}{ll}\text { - } & \text { Restless leg syndrome } \\
\text { - Sleep breathing disorders (apnea, } \\
\text { sleep maintenance insomnia) } \\
\text { Circadian rhythm disorders (phase } \\
\text { advance or delay) }\end{array}$ & $\begin{array}{ll}\text { - } & \text { Sleep breathing disorders } \\
& \text { (apnea) } \\
\text { - } & \text { Narcolepsy } \\
\text { - } & \text { Kleine-Levine syndrome } \\
\text { - } & \text { Behaviorally induced } \\
& \text { insufficient sleep syndrome } \\
\text { - } & \begin{array}{l}\text { Circadian rhythm disorders } \\
\text { (phase advance or delay) }\end{array} \\
\end{array}$ \\
\hline Blood tests & \multicolumn{2}{|c|}{$\begin{aligned} & \text { - } \text { Endocrine (pituitary) function } \\
& \circ \text { Somatotrophins (growth hormone (GH)) } \\
& \circ \text { Corticotropins (adrenocorticotropic hormone (ACTH), beta- } \\
& \text { endorphin) } \\
& \circ \text { Thyrotrophins (thyroid-stimulating hormone (TSH)) } \\
& \circ \text { Lactotrophins (prolactin (PRL)) } \\
& \circ \text { Gonadotropins (luteinizing hormone (LH), follicle-stimulating } \\
& \text { hormone (FSH)) } \\
&\end{aligned}$} \\
\hline INTERVENTIONS & \multicolumn{2}{|c|}{$\begin{array}{l}\text { If sleep-wake disturbances are associated to another psychiatric or medical condition, } \\
\text { this condition should also be treated. }\end{array}$} \\
\hline $\begin{array}{l}\text { Modifications of the } \\
\text { hospital environment }\end{array}$ & \multicolumn{2}{|c|}{$\begin{array}{ll}\text { - } & \text { Ear plugs } \\
\text { - } & \text { Eye mask } \\
\text { - } & \text { Minimizing staff interventions during nighttime/naps } \\
\text { - } & \text { Light therapy or exposure to natural light in the morning (hypersomnia) }\end{array}$} \\
\hline Non-pharmacological & \multicolumn{2}{|c|}{$\begin{array}{ll}\text { - } & \text { Sleep hygiene } \\
\circ & \text { Regular bed/wake times } \\
\circ & \text { Avoiding lengthy daytime naps (more than } 60 \text { minutes) } \\
\circ & \text { Avoiding daytime naps after 3:00 PM } \\
\circ & \text { Avoiding coffee and alcohol } 4 \text { to } 6 \text { hours prior to bedtime } \\
\circ & \text { Avoiding strenuous exercise at least one hour prior to bedtime } \\
\circ & \text { Avoiding stimulant activities at least one hour prior to bedtime (video } \\
& \text { games, suspense movies) } \\
\circ \quad \text { Avoiding nicotine in the hours prior to bedtime or during awakenings } \\
\text { in the night } \\
\circ \quad \text { Avoiding light and noise in the bedroom during the night } \\
\circ \quad \text { Using the bed only for sleep or sexual activities } \\
\circ \quad \text { Going to bed only when ready to sleep } \\
\text { - Light therapy in the morning (hypersomnia) }\end{array}$} \\
\hline
\end{tabular}




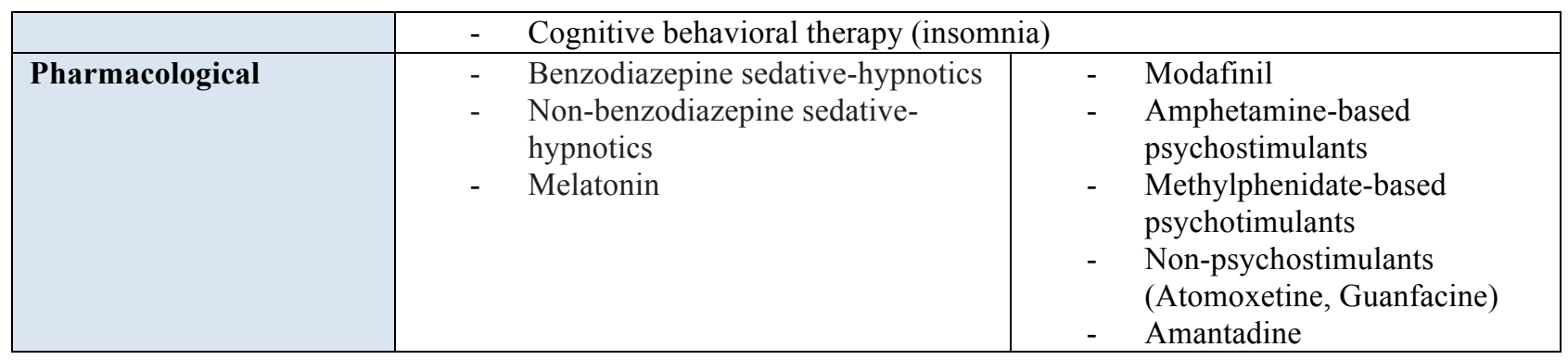

Prescription and over-the-counter pharmacologic interventions are the most common and accessible treatments for insomnia. However, although pharmacologic interventions have been effective in treating sleep disturbances in healthy individuals, it has been consistently reported that benzodiazepines, the most frequently prescribed sleep agent used to treat insomnia, and GABA-agonists result in cognitive impairment when plasma levels are at their peak [86]. There also exists some evidence suggesting that benzodiazepines produce residual effects on cognition, whereas some have shown the GABA agonists may have detrimental effects on neuroplasticity [86]. Moreover, the side-effects of sedative-hypnotic sleep-agents, such as the Z-drugs, include daytime drowsiness, as well as cognitive and psychomotor impairments, all of which are likely to add to the burden of TBI sequelea [87].

Conversely, the use of oral melatonin by critically ill patients has also been shown to improve sleep duration and efficiency [88]. Additionally, melatonin has been shown to have remarkable antioxidant properties, preserves mitochondrial homeostasis, may protect against neurodegenerative processes, and combat free radical damage in the brain, therefore acting as a therapeutic agent in the treatment of cerebral oedema following TBI [89]. However, there appears to be no study that has specifically assessed the efficiency of melatonin to improve sleep and circadian rhythms in TBI patients.

Cognitive behavioral therapy (CBT), including behavioral interventions such as Stimulus Control or Sleep Restriction (restriction of time in bed), has been shown to be efficient in improving the sleep efficiency, reducing sleep onset latency, and reducing the number of nocturnal awakenings, of mild to severe TBI patients suffering from insomnia [90,91]. Strong evidence supports the use of CBT for primary insomnia in the general population, and this 
intervention is being increasingly disseminated in populations with diverse health conditions by non-sleep specialists.

When used in a small sample of patients with TBI (unspecified severity; $\mathrm{n}=12 ; 2.2 \pm 1.3$ years post-injury) and insomnia complaints, acupuncture has also been shown to have a beneficial effect on perception of insomnia severity and improved cognitive functioning, though acupuncture had no effect on sleep time, as measured by actigraphy [92].

\subsection{Interventions to improve hypersomnia}

Prior to treating hypersomnia, as with insomnia, the clinician should screen for medical and psychiatric conditions that may be associated with, or underlie hypersomnia (see Table 1). If sleep comorbidities (e.g. sleep breathing disorders (apnea), periodic limb movement in sleep, and narcolepsy) emerge, usual treatment for these conditions is recommended.

Pharmacological treatments used in the management of hypersomnia include modafinil, amphetamine-based psychostimulants, methylphenidate-based psychostimulants, nonpsychostimulants, and amantadine, though studies on their effectiveness remain inconclusive with regards to post-TBI hypersomnia. For example, while Jha et al. [93] found no difference between modafinil and placebo, Kaiser et al. [94] observed a decrease in post-TBI daytime sleepiness, as measured by the ESS, the Maintenance of Wakefulness Test (MWT), and the amount of time spent awake according to actigraphy. Wiseman-Hakes et al. [57] reported that a methylphenidate-based psychostimulant was successful in improving day-time alertness and cognitive performance in a case study evaluation of an individual with severe TBI and post traumatic hypersomnia.

\section{Conclusions and study perspectives}

The goal of this review was to describe the current state of knowledge on sleep-wake disturbances and circadian rhythm alterations following TBI. We aimed to describe the possible causes of these alterations, and explore the pharmacologic and non-pharmacologic treatment options to treat them. 
Sleep disturbances have been shown to exacerbate trauma related cognitive, communication and mood impairments, as well as pain, and compromise the recovery process $[23,57]$. Memory and new learning are often impaired following TBI of all severities, and the importance of sleep in hippocampal function, learning and the formation of memory in humans has been confirmed by imaging studies [93]. From a more general perspective, partial or chronic sleep restriction has negative repercussions for behavioral, cognitive, inflammatory, immune, cardiovascular, metabolic, and endocrine functions [96-100]. Taken together, these results suggest that acute and chronic sleep restriction and/or fragmentation in TBI survivors can hinder processes of physical, psychological and cognitive recovery, and lead to a reduction in learning capabilities, neural plasticity, and neurogenesis. Interestingly, Wiseman-Hakes et al. [23] reported functional, as well as clinically and statistically significant improvements in sustained and divided attention, working memory, speed of language processing and mood in response to individualized treatment of post-trauma onset sleep-wake disorders in adults (including sleep hygiene recommendations, pharmacological interventions and/or apnea treatment with followup).

Further studies should investigate how these sleep-wake and circadian disturbances originate and evolve, and whether measures can be taken to prevent them. Various treatment options should be further investigated, particularly in the acute phase where recovery is most crucial for the optimisation of patient functionality and quality of life. 


\section{References}

1. Cassidy JD, Carroll LJ, Peloso PM, Borg J, von Holst H, Holm L, et al. Incidence, risk factors and prevention of mild traumatic brain injury: results of the WHO Collaborating Centre Task Force on Mild Traumatic Brain Injury. Journal of rehabilitation medicine : official journal of the UEMS European Board of Physical and Rehabilitation Medicine. 2004 Feb(43 Suppl):2860 .

2. Kraus J CL. Epidemiology. In: Silver JM MT, Yudofsky MD, editor. Textbook of traumatic brain injury. Arlington, VA: American Psychiatric Publishing; 2005.

3. Ouellet MC, Beaulieu-Bonneau S, Morin CM. Insomnia in patients with traumatic brain injury: frequency, characteristics, and risk factors. J Head Trauma Rehabil. 2006 MayJun;21(3):199-212.

4. Castriotta RJ, Wilde MC, Lai JM, Atanasov S, Masel BE, Kuna ST. Prevalence and consequences of sleep disorders in traumatic brain injury. J Clin Sleep Med. 2007 Jun 15;3(4):349-56.

5. Baumann CR. Traumatic brain injury and disturbed sleep and wakefulness. Neuromolecular medicine. 2012 Sep;14(3):205-12.

6. Castriotta RJ, Murthy JN. Sleep disorders in patients with traumatic brain injury: a review. CNS drugs. 2011 Mar;25(3):175-85.

7. Wiseman-Hakes C, Gargaro J, Colantonio A. Sleep and wake disorders following traumatic brain injury: A systematic review of the literature. rit Rev Phys Rehabil Med. 2009;21(34):317-74.

8. Menon DK, Schwab K, Wright DW, Maas AI. Position statement: definition of traumatic brain injury. Archives of Physical Medecine Rehabilitation. 2010 Nov;91(11):1637-40.

9. Congress of Neurological Surgeon. Committe on Head Injury Nomenclature: glossary of head injury. Clin Neurosurg. 1966;12:386-94.

10. Teasdale G, Jennett B. Assessment of coma and impaired consciousness. A practical scale. Lancet. 1974 Jul 13;2(7872):81-4.

11. Popovic JR, Kozak LJ. National hospital discharge survey: annual summary, 1998. Vital Health Stat 13. $2000 \operatorname{Sep}(148): 1-194$.

12. Rutland-Brown W, Langlois JA, Thomas KE, Xi YL. Incidence of traumatic brain injury in the United States, 2003. J Head Trauma Rehabil. 2006 Nov-Dec;21(6):544-8. 
13. Zygun DA, Laupland KB, Hader WJ, Kortbeek JB, Findlay C, Doig CJ, et al. Severe traumatic brain injury in a large Canadian health region. Can J Neurol Sci. 2005 Feb;32(1):87-92.

14. Bruns J, Jr., Hauser WA. The epidemiology of traumatic brain injury: a review. Epilepsia. 2003;44 Suppl 10:2-10.

15. de Guise E, LeBlanc J, Feyz M, Lamoureux J. Prediction of outcome at discharge from acute care following traumatic brain injury. J Head Trauma Rehabil. 2006 Nov-Dec;21(6):527-36.

16. de Guise E, Leblanc J, Feyz M, Lamoureux J. Prediction of the level of cognitive functional independence in acute care following traumatic brain injury. Brain Inj. 2005 Dec;19(13):1087-93.

17. Kosch Y, Browne S, King C, Fitzgerald J, Cameron I. Post-traumatic amnesia and its relationship to the functional outcome of people with severe traumatic brain injury. Brain Inj. 2010;24(3):479-85.

18. LeBlanc J, de Guise E, Gosselin N, Feyz M. Comparison of functional outcome following acute care in young, middle-aged and elderly patients with traumatic brain injury. Brain Inj. 2006 Jul;20(8):779-90.

19. de Guise E, Leblanc J, Dagher J, Lamoureux J, Jishi AA, Maleki M, et al. Early outcome in patients with traumatic brain injury, pre-injury alcohol abuse and intoxication at time of injury. Brain Inj. 2009 Oct;23(11):853-65.

20. McCullagh S, Feinstein A. Cognitive changes. In: Silver J, McAllister T, Yudofsky S, editors. Textbook of traumatic brain injury. Arlington, VA: American Psychiatric publishing, Inc.; 2005. p. 321-35.

21. Riggio S, Wong M. Neurobehavioral sequelae of traumatic brain injury. Mt Sinai J Med. 2009 Apr;76(2):163-72.

22. McAllister T. Mild traumatic brain injury and the postconcussion syndrome. In: Silver J, McAllister T, Yudofsky S, editors. Textbook of traumatic brain injury. Arlington, VA: American Psychiatric publishing, Inc.; 2005. p. 279-308.

23. Wiseman-Hakes C, Murray B, Moineddin R, Rochon E, Cullen N, Gargaro J, et al. Evaluating the impact of treatment for sleep/wake disorders on recovery of cognition and communication in adults with chronic TBI. Brain Inj. 2013;27(12):1364-76. 
24. Lippert-Gruner M, Lefering R, Svestkova O. Functional outcome at 1 vs. 2 years after severe traumatic brain injury. Brain Inj. 2007 Sep;21(10):1001-5.

25. Selassie AW, Zaloshnja E, Langlois JA, Miller T, Jones P, Steiner C. Incidence of long-term disability following traumatic brain injury hospitalization, United States, 2003. J Head Trauma Rehabil. 2008 Mar-Apr;23(2):123-31.

26. Shames J, Treger I, Ring H, Giaquinto S. Return to work following traumatic brain injury: trends and challenges. Disabil Rehabil. 2007 Sep 15;29(17):1387-95.

27. Chaput G, Giguere JF, Chauny JM, Denis R, Lavigne G. Relationship among subjective sleep complaints, headaches, and mood alterations following a mild traumatic brain injury. Sleep Med. 2009 Aug;10(7):713-6.

28. Gosselin N, Lassonde M, Petit D, Leclerc S, Mongrain V, Collie A, et al. Sleep following sport-related concussions. Sleep Med. 2009 Jan;10(1):35-46.

29. Rao V, Bergey A, Hill H, Efron D, McCann U. Sleep disturbance after mild traumatic brain injury: indicator of injury? J Neuropsychiatry Clin Neurosci. 2011 Spring;23(2):201-5.

30. Baumann CR, Werth E, Stocker R, Ludwig S, Bassetti CL. Sleep-wake disturbances 6 months after traumatic brain injury: a prospective study. Brain. 2007 Jul;130(Pt 7):1873-83.

31. Khoury S, Chouchou F, Amzica F, Giguere JF, Denis R, Rouleau GA, et al. Rapid EEG activity during sleep dominates in mild traumatic brain injury patients with acute pain. $\mathrm{J}$ Neurotrauma. 2013 Apr 15;30(8):633-41.

32. Feige B, Baglioni C, Spiegelhalder K, Hirscher V, Nissen C, Riemann D. The microstructure of sleep in primary insomnia: An overview and extension. Int J Psychophysiol. 2013 Aug;89(2):171-80.

33. Beetar JT, Guilmette TJ, Sparadeo FR. Sleep and pain complaints in symptomatic traumatic brain injury and neurologic populations. Arch Phys Med Rehabil. 1996 Dec;77(12):1298-302.

34. Clinchot DM, Bogner J, Mysiw WJ, Fugate L, Corrigan J. Defining sleep disturbance after brain injury. Am J Phys Med Rehabil. 1998 Jul-Aug;77(4):291-5.

35. Collen J, Orr N, Lettieri CJ, Carter K, Holley AB. Sleep disturbances among soldiers with combat-related traumatic brain injury. Chest. 2012 Sep;142(3):622-30.

36. Bryan CJ. Repetitive Traumatic Brain Injury (or Concussion) Increases Severity of Sleep Disturbance among Deployed Military Personnel. Sleep. 2013;36(6):941-6. 
37. Williams BR, Lazic SE, Ogilvie RD. Polysomnographic and quantitative EEG analysis of subjects with long-term insomnia complaints associated with mild traumatic brain injury. Clin Neurophysiol. 2008 Feb;119(2):429-38.

38. Schreiber S, Barkai G, Gur-Hartman T, Peles E, Tov N, Dolberg OT, et al. Long-lasting sleep patterns of adult patients with minor traumatic brain injury (mTBI) and non-mTBI subjects. Sleep Med. 2008 Jul;9(5):481-7.

39. Duclos C, Dumont M, Blais H, Paquet J, Laflamme E, de Beaumont L, et al. Rest-Activity Cycle Disturbances in the Acute Phase of Moderate to Severe Traumatic Brain Injury. Neurorehabil Neural Repair. 2013(in press).

40. Wiseman-Hakes C, Gaudreault P, Duclos C, Bernard F, Carrier J, Gosselin N. Examination of Polysomnography in Hospitalized Patients with Acute Moderate-Severe Traumatic Brain Injury[abstract]. Sleep Med. 2013 (in press).

41. Makley MJ, English JB, Drubach DA, Kreuz AJ, Celnik PA, Tarwater PM. Prevalence of sleep disturbance in closed head injury patients in a rehabilitation unit. Neurorehabil Neural Repair. 2008 Jul-Aug;22(4):341-7.

42. Makley MJ, Johnson-Greene L, Tarwater PM, Kreuz AJ, Spiro J, Rao V, et al. Return of memory and sleep efficiency following moderate to severe closed head injury. Neurorehabil Neural Repair. 2009 May;23(4):320-6.

43. Nakase-Richardson R, Sherer M, Barnett SD, Yablon SA, Evans CC, Kretzmer T, et al. Prospective evaluation of the nature, course, and impact of acute sleep abnormality after traumatic brain injury. Arch Phys Med Rehabil. 2013 May;94(5):875-82.

44. Shekleton JA, Parcell DL, Redman JR, Phipps-Nelson J, Ponsford JL, Rajaratnam SMW. Sleep disturbance and melatonin levels following traumatic brain injury. Neurology. 2010 May 25;74(21):1732-8.

45. Huang W, Bliwise DL, Johnson TM, Long Q, Kutner N, Stringer AY. Correlates of persistent sleep complaints after traumatic brain injury. Neuropsychological rehabilitation. 2013;23(5):698-714.

46. Fogelberg DJ, Hoffman JM, Dikmen S, Temkin NR, Bell KR. Association of sleep and cooccurring psychological conditions at 1 year after traumatic brain injury. Arch Phys Med Rehabil. 2012 Aug;93(8):1313-8. 
47. Parcell DL, Ponsford JL, Rajaratnam SM, Redman JR. Self-reported changes to nighttime sleep after traumatic brain injury. Arch Phys Med Rehabil. 2006 Feb;87(2):278-85.

48. Ponsford JL, Parcell DL, Sinclair KL, Roper M, Rajaratnam SM. Changes in sleep patterns following traumatic brain injury: a controlled study. Neurorehabil Neural Repair. 2013 Sep;27(7):613-21.

49. Cantor JB, Bushnik T, Cicerone K, Dijkers MP, Gordon W, Hammond FM, et al. Insomnia, fatigue, and sleepiness in the first 2 years after traumatic brain injury: an NIDRR TBI model system module study. J Head Trauma Rehabil. 2012 Nov-Dec;27(6):E1-14.

50. Mathias JL, Alvaro PK. Prevalence of sleep disturbances, disorders, and problems following traumatic brain injury: a meta-analysis. Sleep Med. 2012 Aug;13(7):898-905.

51. Verma A, Anand V, Verma NP. Sleep disorders in chronic traumatic brain injury. J Clin Sleep Med. 2007 Jun 15;3(4):357-62.

52. Sommerauer M, Valko PO, Werth E, Baumann CR. Excessive sleep need following traumatic brain injury: a case-control study of 36 patients. J Sleep Res. 2013 Jul 9.Epub 2013/07/11.

53. Ouellet MC, Morin CM. Subjective and objective measures of insomnia in the context of traumatic brain injury: a preliminary study. Sleep Med. 2006 Sep 2006;7(6):486-97.

54. Beaulieu-Bonneau S, Morin CM. Sleepiness and fatigue following traumatic brain injury. Sleep Med. 2012 Jun;13(6):598-605.

55. Watson NF, Dikmen S, Machamer J, Doherty M, Temkin N. Hypersomnia following traumatic brain injury. J Clin Sleep Med. 2007 Jun 15;3(4):363-8.

56. Rao V SJ, Vaishnavi S, Rastogi P, Mielke M, Noll K, Cornwell E, Schretlen D, Makley M. Prevalence and types of sleep disturbances acutely after traumatic brain injury. Brain Inj. 2008 May 2008;22(5):381-6.

57. Wiseman-Hakes C, Victor JC, Brandys C, Murray BJ. Impact of post-traumatic hypersomnia on functional recovery of cognition and communication. Brain Inj. 2011;25(12):1256-65.

58. Masel BE, Scheibel RS, Kimbark T, Kuna ST. Excessive daytime sleepiness in adults with brain injuries. Arch Phys Med Rehabil. 2001 Nov;82(11):1526-32.

59. Mignot E, Lin L, Finn L, Lopes C, Pluff K, Sundstrom ML, et al. Correlates of sleep-onset REM periods during the Multiple Sleep Latency Test in community adults. Brain. 2006 Jun;129(Pt 6):1609-23. 
60. Hartl R, Ghajar J. Neurosurgical Interventions. In: Silver J, McAllister T, Yudofsky S, editors. Textbook of Traumatic Brain Injury. Washington, DC: American Psychiatric Publishing; 2005. p. 51-8.

61. Kumar R, Husain M, Gupta RK, Hasan KM, Haris M, Agarwal AK, et al. Serial changes in the white matter diffusion tensor imaging metrics in moderate traumatic brain injury and correlation with neuro-cognitive function. J Neurotrauma. 2009 Apr;26(4):481-95.

62. Baumann CR, Stocker R, Imhof HG, Trentz O, Hersberger M, Mignot E, et al. Hypocretin-1 (orexin A) deficiency in acute traumatic brain injury. Neurology. 2005 Jul 12;65(1):147-9.

63. Takahashi JS, Zatz M. Regulation of circadian rhythmicity. Science. 1982 Sep 17;217(4565):1104-11.

64. Czeisler CA, Gooley JJ. Sleep and circadian rhythms in humans. Cold Spring Harb Symp Quant Biol. 2007;72:579-97.

65. Barion A, Zee PC. A clinical approach to circadian rhythm sleep disorders. Sleep Med. 2007 Sep;8(6):566-77.

66. Ayalon L, Borodkin K, Dishon L, Kanety H, Dagan Y. Circadian rhythm sleep disorders following mild traumatic brain injury. Neurology. 2007 Apr 3;68(14):1136-40.

67. Ponsford JL, Ziino C, Parcell DL, Shekleton JA, Roper M, Redman JR, et al. Fatigue and sleep disturbance following traumatic brain injury--their nature, causes, and potential treatments. J Head Trauma Rehabil. 2012 May-Jun;27(3):224-33.

68. Steele DL, Rajaratnam SM, Redman JR, Ponsford JL. The effect of traumatic brain injury on the timing of sleep. Chronobiol Int. 2005;22(1):89-105.

69. Coogan AN, Wyse CA. Neuroimmunology of the circadian clock. Brain Res. 2008 Sep 26;1232:104-12.

70. Boone DR, Sell SL, Micci MA, Crookshanks JM, Parsley M, Uchida T, et al. Traumatic brain injury-induced dysregulation of the circadian clock. PLoS ONE. 2012;7(10):e46204.

71. Hardin KA. Sleep in the ICU: potential mechanisms and clinical implications. Chest. 2009 Jul;136(1):284-94.

72. Paul T, Lemmer B. Disturbance of circadian rhythms in analgosedated intensive care unit patients with and without craniocerebral injury. Chronobiol Int. 2007;24(1):45-61.

73. Paparrigopoulos T, Melissaki A, Tsekou H, Efthymiou A, Kribeni G, Baziotis N, et al. Melatonin secretion after head injury: a pilot study. Brain Inj. 2006 Jul;20(8):873-8. 
74. Llompart-Pou JA, Perez G, Raurich JM, Riesco M, Brell M, Ibanez J, et al. Loss of cortisol circadian rhythm in patients with traumatic brain injury: a microdialysis evaluation. Neurocrit Care. 2010 Oct;13(2):211-6.

75. van der Eerden AW, Twickler MT, Sweep FC, Beems T, Hendricks HT, Hermus AR, et al. Should anterior pituitary function be tested during follow-up of all patients presenting at the emergency department because of traumatic brain injury? Eur J Endocrinol. 2010 Jan;162(1):19-28.

76. Bushnik T, Englander J, Katznelson L. Fatigue after TBI: association with neuroendocrine abnormalities. Brain Inj. 2007 Jun;21(6):559-66.

77. Richardson A, Allsop M, Coghill E, Turnock C. Earplugs and eye masks: do they improve critical care patients' sleep? Nurs Crit Care. 2007 Nov-Dec;12(6):278-86.

78. Friese RS Diaz-Arrastia R, McBride D, Frankel H, Gentilello LM. Quantity and quality of sleep in the surgical intensive care unit: are our patients sleeping? J Trauma. 2007 Dec 2007;63(6):1210-4.

79. Tamburri LM, DiBrienza R, Zozula R, Redeker NS. Nocturnal care interactions with patients in critical care units. Am J Crit Care. 2004 Mar;13(2):102-12; quiz 14-5.

80. Dispersyn G, Pain L, Challet E, Touitou Y. General anesthetics effects on circadian temporal structure: an update. Chronobiol Int. 2008 Nov;25(6):835-50.

81. Yetkin S, Aydin H, Ozgen F. Polysomnography in patients with post-traumatic stress disorder. Psychiatry Clin Neurosci. 2010 Jun;64(3):309-17.

82. Khoury S, Gosselin N, Chouchou F, Giguère J-F, Lavigne G. Perturbations du sommeil par la douleur chez les traumatisés crâniens léger. Médecine Sciences Amérique. 2012;2(1):39-50.

83. Fahey CD, Zee PC. Circadian rhythm sleep disorders and phototherapy. Psychiatr Clin North Am. 2006 Dec;29(4):989-1007; abstract ix.

84. Morgenthaler TI, Lee-Chiong T, Alessi C, Friedman L, Aurora RN, Boehlecke B, et al. Practice parameters for the clinical evaluation and treatment of circadian rhythm sleep disorders. An American Academy of Sleep Medicine report. Sleep. 2007 Nov;30(11):144559.

85. Taguchi T, Yano M, Kido Y. Influence of bright light therapy on postoperative patients: a pilot study. Intensive Crit Care Nurs. 2007 Oct;23(5):289-97. 
86. Larson EB, Zollman FS. The effect of sleep medications on cognitive recovery from traumatic brain injury. J Head Trauma Rehabil. 2010 Jan-Feb;25(1):61-7.

87. Orff HJ, Ayalon L, Drummond SPA. Traumatic brain injury and sleep disturbance: a review of current research. J Head Trauma Rehabil. 2009 May-Jun;24(3):155-65.

88. Bourne RS, Mills GH, Minelli C. Melatonin therapy to improve nocturnal sleep in critically ill patients: encouraging results from a small randomised controlled trial. Crit Care. 2008;12(2):R52.

89. Pandi-Perumal SR, BaHammam AS, Brown GM, Spence DW, Bharti VK, Kaur C, et al. Melatonin antioxidative defense: therapeutical implications for aging and neurodegenerative processes. Neurotoxicity research. 2013 Apr;23(3):267-300.

90. Ouellet MC, Morin CM. Cognitive behavioral therapy for insomnia associated with traumatic brain injury: a single-case study. Arch Phys Med Rehabil. 2004 Aug;85(8):1298-302.

91. Ouellet MC, Morin CM. Efficacy of cognitive-behavioral therapy for insomnia associated with traumatic brain injury: a single-case experimental design. Arch Phys Med Rehabil. 2007 Dec;88(12):1581-92.

92. Zollman FS, Larson EB, Wasek-Throm LK, Cyborski CM, Bode RK. Acupuncture for treatment of insomnia in patients with traumatic brain injury: a pilot intervention study. $\mathrm{J}$ Head Trauma Rehabil. 2012 Mar-Apr;27(2):135-42.

93. Jha A, Weintraub A, Allshouse A, Morey C, Cusick C, Kittelson J, et al. A randomized trial of modafinil for the treatment of fatigue and excessive daytime sleepiness in individuals with chronic traumatic brain injury. J Head Trauma Rehabil. 2008 Jan-Feb;23(1):52-63.

94. Kaiser PR, Valko PO, Werth E, Thomann J, Meier J, Stocker R, et al. Modafinil ameliorates excessive daytime sleepiness after traumatic brain injury. Neurology. 2010 Nov $16 ; 75(20): 1780-5$.

95. Orban P, Rauchs G, Balteau E, Degueldre C, Luxen A, Maquet P, et al. Sleep after spatial learning promotes covert reorganization of brain activity. Proc Natl Acad Sci U S A. 2006 May 2;103(18):7124-9.

96. Goel N, Rao H, Durmer JS, Dinges DF. Neurocognitive consequences of sleep deprivation. Semin Neurol. 2009 Sep;29(4):320-39. 
97. Shamsuzzaman AS, Winnicki M, Lanfranchi P, Wolk R, Kara T, Accurso V, et al. Elevated C-reactive protein in patients with obstructive sleep apnea. Circulation. 2002 May 28;105(21):2462-4.

98. Spiegel K, Sheridan JF, Van Cauter E. Effect of sleep deprivation on response to immunization. JAMA. 2002 Sep 25;288(12):1471-2.

99. Mullington JM, Haack M, Toth M, Serrador JM, Meier-Ewert HK. Cardiovascular, inflammatory, and metabolic consequences of sleep deprivation. Prog Cardiovasc Dis. 2009 Jan-Feb;51(4):294-302.

100. Spiegel K, Leproult R, Van Cauter E. Impact of sleep debt on metabolic and endocrine function. Lancet. 1999 Oct 23;354(9188):1435-9. 